\title{
Magnetic scattering and superconductivity in $\mathrm{Nd}_{1 \cdot 86} \mathrm{Ce}_{0.14} \mathrm{CuO}_{4-y}$
}

\author{
V VIJAYASHREE, C K SUBRAMANIAM and R SRINIVASAN \\ Department of Physics, Indian Institute of Technology, Madras 600036, India
}

\begin{abstract}
Nd}_{1 \cdot 86} \mathrm{Ce}_{0 \cdot 14} \mathrm{CuO}_{4-y}$ is superconducting below about $20 \mathrm{~K}$ and electrons are considered to be responsible for superconductivity in these materials as in the case of $\mathrm{Ln}_{2-x} \mathrm{Ce}_{x} \mathrm{CuO}_{4-y}$ and $\mathrm{Ln}_{2-x} \mathrm{Th}_{x} \mathrm{CuO}_{4-y}$. Structurally these materials are not very different from the p-type superconductors $\mathrm{La}_{z}{ }_{x} \mathrm{Sr}_{x} \mathrm{CuO}_{4}$. In both these types of superconductors, the parent compounds are antiferromagnetically-ordered insulators. The induction of holes or electrons by substitution destroys magnetic interactions and brings about superconductivity. Peng and coworkers have studied the resistivity variation of both superconducting and nonsuperconducting $\mathrm{Nd}_{1.85} \mathrm{Ce}_{0.15} \mathrm{CuO}_{4-y}$ and have found a decrease in resistivity with temperature, obeying a $\ln T$ dependence in the superconducting samples. Such a variation was not seen by them in the nonsuperconducting samples. They ascribe the $\ln T$ variation seen in their superconducting samples to arise from magnetic scattering of electrons. To study whether such an effect exists, $\mathrm{Nd}_{1 \cdot 86} \mathrm{Ce}_{0-14} \mathrm{CuO}_{4-y}$ was subjected to various annealing conditions and the resistivity behaviour is presented here. Our results differ from those of Peng et al.
\end{abstract}

Keywords. Electron superconductors; magnetic scattering; $\ln T$ variation; resistivity.

\section{Introduction}

The discovery of superconductivity in $\mathrm{Nd}_{1 \cdot 85} \mathrm{Ce}_{0 \cdot 15} \mathrm{CuO}_{4-y}$ has generated a lot of enthusiasm. This has shown that superconductivity in the $\mathrm{Cu}-\mathrm{O}$ based superconductors can arise both due to electrons and holes. The parent compound $\mathrm{La}_{2} \mathrm{CuO}_{4}$ for $p$-type superconductors and $\mathrm{Nd}_{2} \mathrm{CuO}_{4}$ for $n$-type superconductors have structural similarities. Both of them are antiferromagnetically-ordered insulators. Partial substitution of $\mathrm{Sr} / \mathrm{Ba}$ for $\mathrm{La}$ in $\mathrm{La}_{2} \mathrm{CuO}_{4}$ or $\mathrm{Ce} / \mathrm{Th}$ for $\mathrm{Nd}$ in $\mathrm{Nd}_{2} \mathrm{CuO}_{4}$ kill antiferromagnetic ordering and induces superconductivity.

Peng et al (1989) interpreted $\ln T$ dependence of resistivity found by them in their superconducting samples as arising from magnetic scattering of electrons. Such a $\ln T$ term was not found by them in the non-superconducting samples. On the other hand, resistivity studies on single crystals by Tarascon et al (1989) and Hidaka and Suzuki (1989) have shown that resistivity increases as temperature increases, both along and perpendicular to the $a-b$ plane. It is, therefore, possible that this $\ln T$ dependence may not be intrinsic to the material but may arise from intergranular semiconducting layers. In order to find whether such a $\ln T$ dependence exists, and its dependence on annealing conditions, we have carried out systematic studies on $\mathrm{Nd}_{1.86} \mathrm{Ce}_{0 \cdot 14} \mathrm{CuO}_{4-y}$. These are reported in this paper.

\section{Experimental details}

Stoichiometric proportions of high purity $(>4 \mathrm{~N}) \mathrm{Nd}_{2} \mathrm{O}_{3}, \mathrm{CeO}_{2}$ and $\mathrm{CuO}$ were mixed thoroughly and heated in air for $24 \mathrm{~h}$ at $900^{\circ} \mathrm{C}$. The reacted powder was ground well, pressed into pellets and sintered at $1050^{\circ} \mathrm{C}$ for $16 \mathrm{~h}$. Figure 1 shows the XRD of the material. All the peaks of the XRD can be indexed for the tetragonal structure of the 


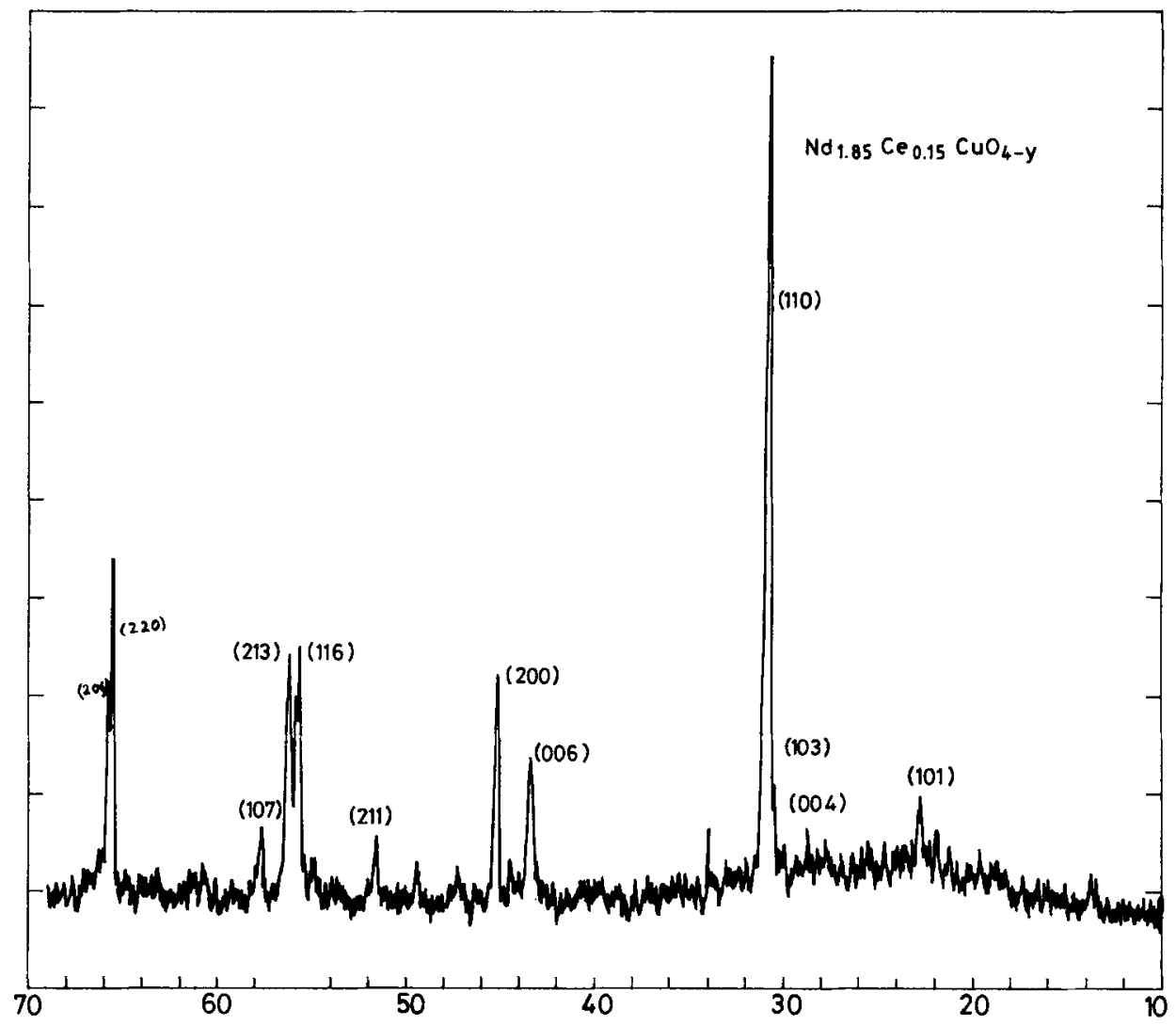

Figure 1. XRD of $\mathrm{Nd}_{1 \cdot 86} \mathrm{Ce}_{0 \cdot 14} \mathrm{CuO}_{4-\gamma}$

single phase compound $\mathrm{Nd}_{1.86} \mathrm{Ce}_{0.14} \mathrm{CuO}_{4-y}$. The lattice parameters are: $a=3.958 \AA$ and $c=12.097 \AA$. The as-prepared sample was not superconducting and it was subjected to the annealing treatments described below to induce superconductivity:

(a) The pellet was heated at $900^{\circ} \mathrm{C}$ for $12 \mathrm{~h}$ in flowing $\mathrm{He}$.

(b) The pellet was heated at $850^{\circ} \mathrm{C}$ for $2 \mathrm{~h}$ under continuous evacuation at a pressure of $10^{-5}$ torr and then slow-cooled to room temperature.

(c) The same procedure as in (b) except that the duration of heating under vacuum was for $6 \mathrm{~h}$.

The resistivity of the samples (a), (b) and (c) were measured by the conventional fourprobe technique from room temperature down to $4 \cdot 2 \mathrm{~K}$.

\section{Results and discussion}

Figure 2 shows the temperature variation of resistivity of samples (a), (b) and (c). Sample (a) was not superconducting down to $4.2 \mathrm{~K}$. Sample (b) showed a steep drop in resistivity at $28.3 \mathrm{~K}$. However, the resistivity at $4.2 \mathrm{~K}$ was still $50 \mu \mathrm{ohm}-\mathrm{cm}$. Sample (c) showed a superconducting onset at $25.8 \mathrm{~K}$ and a zero resistance at $15 \cdot 1 \mathrm{~K}$. We plotted 


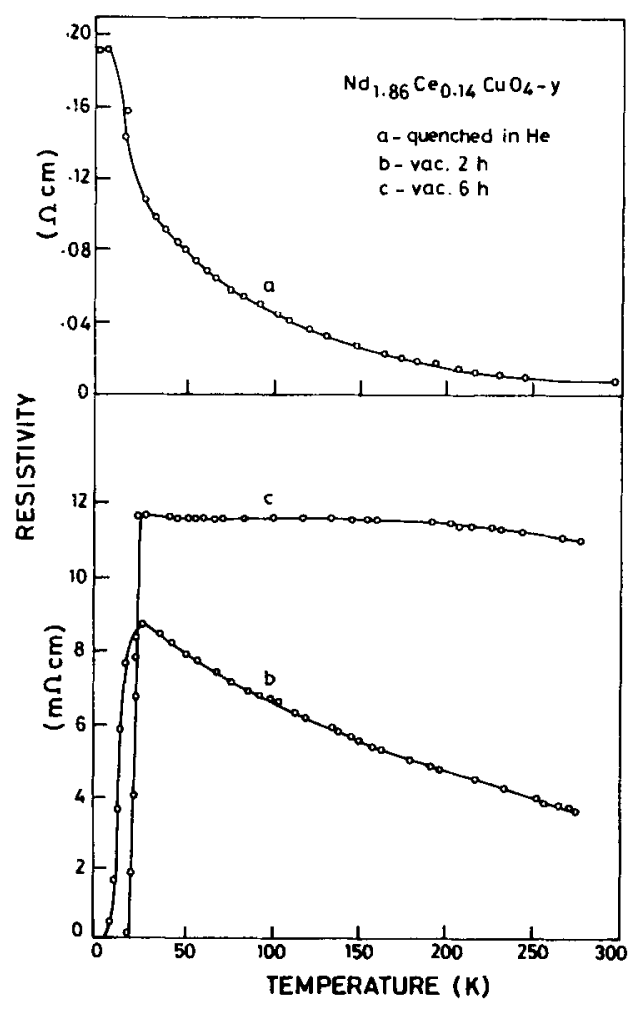

Figure 2. Resistivity versus temperature of $\mathrm{Nd}_{1 \cdot 86} \mathrm{Ce}_{0.14} \mathrm{CuO}_{4-y}$.

resistivity versus $\ln T$ for all these samples. Figure 3 shows such a plot. It is seen that there is a temperature region in which the resistivity shows a logarithmic dependence. Writing,

$$
\rho=\rho_{0}(1-\alpha \ln T)
$$

the values of $\rho_{0}$ and $\alpha$ are collected in table 1 for all these samples. In table 1 , we also give the temperature range over which this relation is valid. It is seen that the coefficient $\alpha$ decreases from sample (a) to sample (c). For the two samples (a) and (b), $\alpha$ is around $0 \cdot 15$, but it goes to zero for sample (c). This indicates that the strength of the $\ln T$ term is more for the non-superconducting samples, a result which contrasts with the result of Peng et al (1989).

Since doing this work, we have carried out similar studies for Ce concentrations $0 \cdot 15$, 0.16 and 0.18 . For the first two compositions, the annealing treatment was varied to induce superconductivity. The sample with Ce concentration 0.18 showed a metallic behaviour. The samples with $C e$ concentrations 0.15 and 0.16 again showed a $\ln T$ behaviour and the parameters $\rho_{0}$ and $\alpha$ are also collected in table 1 (Vijayashree et al 1990). It is again seen that in all the compositions, the strength of the $\ln T$ term decreases as superconductivity is induced in these samples.

If the $\ln T$ term arises from magnetic scattering, these results indicate that superconductivity sets in only if the magnetic scattering becomes weak. On the other hand, one cannot rule out the possibility that this $\ln T$ term arises from a semiconduct- 


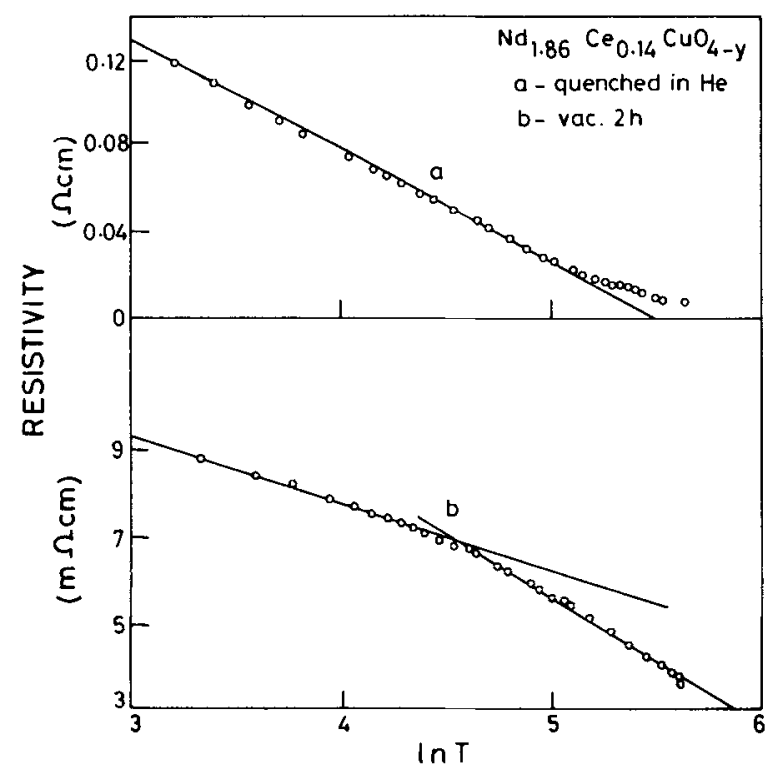

Figure 3. Logarithmic temperature dependence of resistivity of $\mathrm{Nd}_{1 \cdot 86} \mathrm{Ce}_{0 \cdot 14} \mathrm{CuO}_{4-y}$.

Table 1. Parameters in the fit to equation (1) of the temperature dependence of the resistivity of the samples of $\mathrm{Nd}_{2-x} \mathrm{Ce}_{x} \mathrm{CuO}_{4-y}$

\begin{tabular}{|c|c|c|c|c|c|c|}
\hline \multirow{2}{*}{$\begin{array}{c}\text { Sample } \\
\text { no. }\end{array}$} & \multirow[b]{2}{*}{$x$} & \multirow{2}{*}{$\begin{array}{c}\text { Annealed } \\
\text { in }\end{array}$} & $\rho_{\mathrm{RT}}$ & $\rho_{0}$ & \multirow[b]{2}{*}{$\alpha$} & \multirow{2}{*}{$\begin{array}{c}\text { Temperature } \\
\text { range } \\
\text { (in K) }\end{array}$} \\
\hline & & & \multicolumn{2}{|c|}{ (in milli ohm-cm) } & & \\
\hline $\mathbf{a}$ & $0 \cdot 14$ & Helium & 6.95 & $285 \cdot 7$ & $0 \cdot 182$ & $25-150$ \\
\hline \multirow[t]{2}{*}{$\mathrm{b}$} & $0 \cdot 14$ & Vacuum & 3.65 & $\int 14 \cdot 77$ & $0 \cdot 120$ & $36-93$ \\
\hline & & $2 \mathrm{~h}$ & & $21 \cdot 32$ & $0 \cdot 147$ & $121-276$ \\
\hline \multirow[t]{2}{*}{ c } & 0.14 & Vacuum & 11.00 & 11.50 & $\sim 0$ & $30-220$ \\
\hline & (sc) & $6 \mathrm{~h}$ & & & & \\
\hline a & 0.15 & Oxygen & 114.9 & 15,560 & $0 \cdot 188$ & $50-150$ \\
\hline $\mathrm{b}$ & 0.15 & Argon & $16 \cdot 7$ & $102 \cdot 7$ & $0 \cdot 149$ & $47-273$ \\
\hline \multirow[t]{2}{*}{ c } & 0.15 & Vacuum & $2 \cdot 47$ & 3.78 & 0.069 & $70-140$ \\
\hline & (sc) & $6 \mathrm{~h}$ & & & & \\
\hline a & 0.16 & - & $30 \cdot 1$ & 1,154 & $0 \cdot 184$ & $25-150$ \\
\hline b & 0.16 & $\begin{array}{l}\text { Vacuum } \\
2 \mathrm{~h}\end{array}$ & $3 \cdot 0$ & $3 \cdot 165$ & 0.054 & $27-95$ \\
\hline c & 0.16 & $\begin{array}{l}\text { Vacuum } \\
6 \mathrm{~h}\end{array}$ & 6.97 & 6.97 & $\sim 0$ & $27-278$ \\
\hline
\end{tabular}


ing layer at the grain boundaries. If so, the decrease in $\alpha$ as superconductivity is induced must be interpreted as a change in the intergranular boundary from semiconducting to metallic.

\section{Conclusions}

Resistivity has been studied on single-phase samples of $\mathrm{Nd}_{1.86} \mathrm{Ce}_{0.14} \mathrm{CuO}_{4-y}$, subjected to various annealing treatments. There is a temperature region in which the resistivity of the non-superconducting samples decreases linearly with $\ln T$. Measurements for this concentration of $\mathrm{Ce}$ and for other concentrations of Ce indicate clearly that superconductivity sets in only when the coefficient of the $\ln T$ term is reduced. These results are in disagreement with the results of Peng et al (1989). These results may either indicate that superconductivity is seen when the intergranular semiconducting layers become metallic or when the magnetic scattering is suppressed.

\section{Acknowledgements}

The authors are thankful to the Programme Management Board (Superconductivity), Department of Science and Technology, Government of India, for financial support. VV is thankful to the Council of Scientific and Industrial Research for the award of a fellowship.

\section{References}

Hidaka Y and Suzuki M 1989 Nature (London) 338635

Peng J L, Shelton R N and Radousky H B 1989 (Preprint)

Tarascon J M et al 1989 Phys. Rev. B40 4494

Vijayashree R, Subramaniam C K, Sankaranarayanan V, Subba Rao G V and Srinivasan R 1990 Physica C16753 